\title{
Microstructure design for blended feedstock and its thermal durability in lanthanum zirconate based thermal barrier coatings
}

\author{
Dowon Song ${ }^{1}$, Ungyu Paik ${ }^{1, *}$, Xingye Guo ${ }^{2}$, Jing Zhang ${ }^{2}$, Ta-Kwan Woo ${ }^{3}$, Zhe $\mathrm{Lu}^{4}$, \\ Sung-Hoon Jung ${ }^{4}$, Je-Hyun Lee ${ }^{4}$, Yeon-Gil Jung, ${ }^{4, *}$ \\ ${ }^{1}$ Department of Energy Engineering, Hanyang University, Seoul 133-791, \\ Republic of Korea \\ ${ }^{2}$ Department of Mechanical Engineering, Indian University-Purdue University Indianapolis, \\ Indianapolis, IN 46202, USA \\ ${ }^{3}$ Sung-il Turbine Co., Ltd, Busan 618-818, Republic of Korea \\ ${ }^{4}$ School of Materials Science and Engineering, Changwon National University, \\ Changwon, Gyeongnam 641-773, Republic of Korea \\ *Corresponding author. Tel: +82-2-2220-0502, Fax: +82-2-2281-0502 \\ E-mail: upaik@hanyang.ac.kr \\ *Corresponding author. Tel: +82-55-213-3712, Fax: +82-55-262-6486 \\ E-mail: jungyg@changwon.ac.kr
}




\section{Abstract}

The effects of microstructure design on the lifetime performance of lanthanum zirconate $\left(\mathrm{La}_{2} \mathrm{Zr}_{2} \mathrm{O}_{7}\right.$; LZO)-based thermal barrier coatings (TBCs) were investigated through various thermal exposure tests, such as furnace cyclic thermal fatigue, thermal shock, and jet engine thermal shock. To improve the thermal durability of LZO-based TBCs, composite top coats using two feedstock powders of LZO and $8 \mathrm{wt} \%$ yttria-doped stabilized zirconia (8YSZ) were prepared by mixing in different volume ratios (50:50 and 25:75, respectively). In addition, buffer layers were introduced in layered LZO-based TBCs deposited using an airplasma spray method. The TBC with the double buffer layer showed the best thermal cycle performance among all samples in all tests. For applications with relatively slow cooling rates, the thermal durability in single-layer TBCs is more effectively enhanced by controlling a composition ratio in the blended powder, better than introducing a single buffer layer. For applications with relatively fast cooling rates, the thermal durability can be effectively improved by introducing a buffer layer than controlling a composition in the top coat, since the buffer layer provides fast localized stress relief due to its high strain compliance. These research findings allow us to control the TBC structure, and the buffer layer is efficient in improving thermal durability in cyclic thermal environments.

Keywords: Thermal barrier coating; Lanthanum zirconate; Layer structure; Composite; Cyclic thermal exposure. 


\section{Introduction}

Thermal barrier coatings (TBCs) have been widely used to protect the hot-section components of gas turbines and accommodate the increase in turbine inlet temperature, resulting in the improvement of the performance efficiency [1-4]. Most TBC systems consist of a superalloy substrate, an adhesive metallic bond coat, and a thermally insulating ceramic top coat. The typical top coat material in TBC is 7-8 wt\% yttria-doped stabilized zirconia (78YSZ), which is deposited by air-plasma spray (APS) or electron-beam physical vapor deposition [5]. However, the use of YSZ has limitations in satisfying the demand for higher efficiency in gas turbines. At higher operating temperatures, YSZ-based TBCs show a degradation by phase transition and microstructure densification. YSZ transforms from the $t$ ' phase to the tetragonal and cubic phases ( $t$ and $c$ phases, respectively) during the cooling process after operating temperatures above $1200{ }^{\circ} \mathrm{C}$, and then to the monoclinic $(\mathrm{m})$ phase with a volume expansion of about 3-5 vol\% in further operation, resulting in the spallation or delamination of TBCs [6]. In addition, the poor sintering resistance of YSZ at higher temperatures reduces porosity in the top coat, deteriorating the thermal insulation ability of 
reasons.LZO, which has a pyrochlore crystal structure, contains more oxygen vacancies than fluorite YSZ material, resulting in disturbance of lattice scattering, which is the dominant mechanism of thermal conduction in ceramic materials $[6,13]$. The lattice scattering is also interrupted by the atomic weight difference between lanthanum and zirconium, which are 138 and $91 \mathrm{~g} \cdot \mathrm{mol}^{-1}$, respectively. On the other hand, LZO is very stable and has no phase transition in the temperature range of $30-1400^{\circ} \mathrm{C}[14]$.

Although the promising characteristics of LZO, however currently, the biggest challenge of LZO for applications is the relatively poor lifetime performance compared with typical YSZ-based TBCs. LZO has lower coefficient of thermal expansions (CTEs, 9.1$9.7 \times 10^{-6} \cdot \mathrm{K}^{-1}$ for LZO and $10.5-11.5 \times 10^{-6} \cdot \mathrm{K}^{-1}$ for $8 \mathrm{YSZ}$ ) and inferior fracture toughness than YSZ ( $1.1 \mathrm{MPa} \cdot \mathrm{m}^{1 / 2}$ for LZO and $~ 2.0 \mathrm{MPa} \cdot \mathrm{m}^{1 / 2}$ for 8YSZ) $[6,15]$. The former results in severe CTE mismatch with typical bond coat materials. During operation, the delamination of LZO-based TBC was observed due to the stresses at the interface between the top and bond coats that are generated by CTE mismatch [14]. Besides, its low fracture toughness can’t hinder crack propagation and delamination during operation [7, 16]. Vaßen et al. [17] performed a thermal cyclic test to evaluate the improvement of thermal durability by designing double-layer TBCs with 8YSZ layer and LZO outer layer in the range of temperature from $1200{ }^{\circ} \mathrm{C}$ to $1450{ }^{\circ} \mathrm{C}$. Double-layer TBCs showed an enhanced thermal durability compared with LZO single-layer TBC and similar results to an single-layer 8YSZ system at temperatures below $1300^{\circ} \mathrm{C}[17]$. Wang et al. [18] compared the thermal shock (TS) behavior with the single-ceramic-layer 8YSZ and double-ceramic-layer LZO/8YSZ coatings. The double-ceramic-layer coating had a longer TS lifetime even than the single-ceramic-layer coating, indicating the double-layer structure is an efficient design to overcome shortcoming 
of LZO-based TBC [18]. On the other hand, Wang et al. [19] also studied the thermal cyclic behavior of TBC systems with graded coating, the single-layer and double-layer coatings. The thermal cyclic lifetime of the graded coating was certainly extended over that of the single- and double-layer coatings [19].

In this research, a new microstructure of LZO-based TBC was designed with the concept of layer structure using blended LZO and 8YSZ feedstock powders. The mixed LZO and 8YSZ powders with selected volume ratios allow to combine advantages of LZO thermal insulating performance and 8YSZ thermal durability. Moreover, buffer layers, a thin coating layer between the top and bond coats, with the single and double structures were introduced between bond coat and blended top coat to enhance the interfacial stability through compositional continuity. Also, the buffer layer can reduce stresses generated at the interface due to the CTE mismatch between the bond and top coats. The effects of architecture design on the thermal durability were investigated through furnace cyclic thermal fatigue (FCTF), TS, and jet engine thermal shock (JETS) tests. In addition, the microstructure evolution and mechanical properties were observed and evaluated before and after thermal exposure tests. The thermal durability with structure design is discussed, based on a comparison of microstructure evolution and mechanical properties before and after thermal exposure tests.

\section{Experimental procedure}

\subsection{Sample preparation}

The disk-shaped Ni-based superalloy (Nimonic 263, nominal composition of Ni20Cr-20Co-5.9Mo-0.5Al-2.1Ti-0.4Mn-0.3Si-0.06C, in wt\%, ThyssenKrupp VDM, Germany) was used as a substrate. The dimensions of the specimens were $25 \mathrm{~mm}$ in diameter and $5 \mathrm{~mm}$ in thickness. Before deposition of the bond coat, sandblasting was performed on the surface 
of the substrate using $\mathrm{Al}_{2} \mathrm{O}_{3}$ powder with a particle size of about $\sim 420 \mu \mathrm{m}$. The feedstock powder for the bond coat was AMDRY 962 (Sulzer Metco Holding AG, Switzerland, nominal composition of Ni-22Cr-10Al-1.0Y in wt\% and particle size of 56-106 um). METCO $204 \mathrm{C}-$ NS (Sulzer Metco Holding AG, Switzerland, particle size of $45-125 \mu \mathrm{m}$ ) was employed as a feedstock powder for 8YSZ. LAO-109-1 (Praxair Surface Technologies, Indianapolis, IN) was the powder used to produce the LZO coatings. LZO and 8YSZ powders with different volume ratios of 50:50 and 25:75, respectively, were mechanically mixed using a ball mill drive (NITBMT0202, Nano Korea Intec. Ltd., Republic of Korea) for 4 h, and then used as a starting powders.

The thicknesses of the bond and top coats were approximately 150 and $430 \mu \mathrm{m}$, respectively. First, the bond coat was deposited using a commercial APS coating method (9MB, Sulzer Metco, Switzerland), then the top coats including buffer layers were coated using the same equipment. The detailed designs are illustrated in Fig. 1. Two types of singlelayer top coat (SLC) without a buffer layer were deposited using different blended compositions of 50:50 vol\% and 25:75 vol\% in LZO and 8YSZ as shown in Figs. 1(A) and 1(C), respectively. In addition, two types of double-layer top coat (DLC) with a buffer layer were deposited using the 8YSZ and blended powers (Figs. 1(B) and 1(D)). In the DLC TBCs, the buffer layers are different. One (Fig. 1(B)) has only 8YSZ buffer layer and the other (Fig. 1(D)) has two buffer layers of 8YSZ and a blended composition of 25:75 vol\% in LZO and 8YSZ, respectively.

\subsection{Characterizations}

To obtain both cross-sectional microstructure and mechanical properties before and after thermal exposure tests, each TBC sample was cold-mounted using epoxy resin. The 
samples mounted in a fluid epoxy resin were polished using silicon carbide paper and polished using the 3 and $1 \mu \mathrm{m}$ diamond pastes. The cross-sectional microstructure of TBC specimens was observed using a scanning electron microscope (SEM, JEOL Model JSM5610, Japan). The mechanical properties were characterized by two kinds of indentation method, nano-indentation and micro-indentation. Localized elastic modulus, $E$, and hardness, $H$, were evaluated using a nano-indenter (Nano Instruments, MTS Systems Corp., Eden Prairie, USA) with a Berkovich tip (radius of tip $<100 \mathrm{~nm}$ ). The data were selected after indentation up to $50 \mathrm{gf}(0.49 \mathrm{~N})$. The global $\mathrm{H}$ values of the bond and top coats were determined using a micro-indenter (HM-114, Mitutoyo Corp., Japan) with a Vickers tip for a load of $3 \mathrm{~N}$ and holding for $15 \mathrm{~s}$. To obtain reliable values, more than 10 points were tested for each Vickers hardness result. The size of the indentation impression was measured using SEM and all indentations were performed at room temperature.

\subsection{Thermal exposure tests}

A bottom-loading programmable cyclic furnace was used for FCTF tests. The tests were performed at a surface temperature of $1100^{\circ} \mathrm{C}$ with a temperature difference of $150^{\circ} \mathrm{C}$ between the top and bottom surfaces of sample with a dwell time of $40 \mathrm{~min}$, and then natural air cooling was allowed for $20 \mathrm{~min}$ at room temperature. The failure criterion was defined as $25 \%$ buckling or spallation of the top coat in FCTF tests. TS tests were carried out till delamination in a muffle furnace. When the temperature of the furnace reached $1100^{\circ} \mathrm{C}$, the samples were placed in the furnace. In TS tests, the samples were held for $40 \mathrm{~min}$ in the furnace and then directly quenched into water. Throughout the TS, the water temperature was between 20 and $35^{\circ} \mathrm{C}$. More than $50 \%$ of the region spalled in the topcoat or cracked at the interface between the top and bond coats was adopted as the criterion for failure in water- 
quenched samples. In addition, to investigate the thermal cycling performance of TBCs, JETS tests were conducted with using specially designed system; the center of the top coat was exposed for $20 \mathrm{~s}$ to a flame with a temperature of $1400{ }^{\circ} \mathrm{C}$ and directly quenched by nitrogen gas for $20 \mathrm{~s}$, followed by ambient cooling for $1 \mathrm{~min}$. The failure criteria used in JETS tests were typically more than $50 \%$ spallation of the top coat or cracked at the interface between the top and bond coats. At least three samples were tested for each condition.

\section{Results and discussion}

\subsection{Characterization of as-coated samples}

Cross-sectional microstructures and element analysis results of as-coated TBCs are shown in Fig. 2. The bond coat showed a typical microstructure prepared using the APS method in all samples, which contains oxide materials and some pores. The top coat also showed intrinsic defects, such as pores, unmelted particles, and splat boundaries. The top and bond coats were well deposited with controlled thicknesses of $350 \pm 80$ and $140 \pm 20 \mu \mathrm{m}$ in the top and bond coats, respectively, even though the sample C (Fig. 2(C-1)) shows a slightly thicker top coat than others. The thickness of the sample $C$ is about $430 \mu \mathrm{m}$ and the others are in a range of 300-400 $\mu$ m, despite being same deposition parameters. Actually, the thickness difference was not intended. A thick coating thickness may cause the thermal and residual stresses, but the implication was negligible on thermal durability according to previous study [20]. Figures 2(A) and 2(C) are the SLC TBCs using the blended feedstock powders with volume ratios 50:50 and 25:75 of LZO and YSZ, respectively. The as-prepared microstructures corresponded well with the coating design shown in Fig. 1. Figure 2(A-1) shows more LZO content (brighter area) and less YSZ content (darker area) than Fig. 2(C-1). A porous YSZ buffer layer (the first buffer layer) could be observed in both DLC TBCs of 
Figs. 2(B-1) and 2(D-1). The first buffer layer (8YSZ buffer layer) was deposited with a thickness of $60 \mu \mathrm{m}$. In the DLC TBC of Fig. 2(D-1), the second buffer layer with a volume ratio 25:75 of LZO and YSZ, respectively, was inserted between the top coat and the first buffer layer with a thickness of $60 \mu \mathrm{m}$. The TBCs did not show any delamination or cracking at the interface between the top and bond coats. All of the samples showed an irregular surface roughness at the interface. In addition, an indistinguishable interface without obvious large cracks was observed within the top coats, indicating that minimal thermal and residual stresses were imposed at the interfaces.

The $E$ and $H$ values of each TBC from nano-indentation are summarized in Fig. 2. The average imprint sizes in nano-indentation were 9.5, 9.6, and $14.6 \mu \mathrm{m}$ for the top coat, the buffer layer, and the bond coat, respectively. In this study, the layered structures with the buffer layer using the 8YSZ feedstock and blended powders were designed. Therefore, the mechanical properties were evaluated through nano-indentation to verify the compositional continuity or homogeneity with microstructure design, even though the data were from localized regions. The nano-indentation was conducted diagonally from the interface of the bond coat and the substrate to the top coat, with an average gap of $45 \mu \mathrm{m}$. Each interface has been marked as different lines in Figs. 2 (A-2)-(D-2). The dotted black, dotted blue, solid black, solid blue lines indicate the interfaces of the top and bond coats, the top coat and the first buffer layer, the top coat and the second buffer layer, and the second and first buffer layers, respectively. The obtained $E$ and $H$ values within the top coat including the buffer were not significantly different in all samples, indicating that the blended powders were homogeneously deposited. Discontinuity was observed at the interface between the bond and top coats, no matter with or without the buffer layer, especially in the sample B (Fig. 2(B-2)). However, the continuity or discontinuity of mechanical properties, $\mathrm{E}$ and $\mathrm{H}$, at the interface 
between the top and bond coats was a negligible for the lifetime performance of TBC systems in thermal exposure tests, as shown in Table 1.

3.2 Furnace cyclic thermal fatigue test

Equivalent operating hour $(E O H)$ is commonly used for estimating lifetime in inspection of gas turbine system. $E O H$ is a function of the actual operating hours $(A O H)$, coefficient of correction $\left(\sum S_{i}\right)$, load rejection $\left(\sum L R_{i}\right)$, equivalent hours for startup, trips $\left(\sum T_{i}\right)$, rapid load change $\left(\sum L C_{i}\right)$, standby, fuel operation, etc. $[19,21]$. Based on the $E O H$, the conservation and inspection interval units are used rather than actual running hours:

$$
E O H=A O H+20\left[\sum \mathrm{S}_{\mathrm{i}}+\sum L R_{i}+\sum T_{i}+\sum L C_{i}\right] \times F
$$

where $F$ represents the fuel factor (gas: 1.0). In this study, $E O H$ was estimated as an approximate value by multiplying the number of cycles by 21 .

FCTF tests were performed for each sample to evaluate the thermal durability of newly designed TBCs for 1143 cycles $(24,000 \mathrm{EOH})$. The numbers of cycle-to-failure and surface status are summarized in Table 1 . The cross-sectional microstructures of each sample after FCTF tests are shown in Fig. 3. The numbers of cycle-to-failure corresponding to each figure are shown inside each figure. The sample A (Fig. 3(A)) with 50:50 volume ratio composite top coat was delaminated in a range of 106-540 cycles (2,226-11,340 EOH), showing the poorest thermal fatigue resistance. Delamination in the samples B and C occurred in ranges of 270-768 and 653-936 cycles (5,670-16,120 and 13,713-19,650 EOH, respectively), respectively. Enhanced number of cycles in the sample $C$ that contains controlled ratio of LZO and YSZ powders suggests that the composition of the top coat could have more beneficial results on the coating performance than a buffer layer. FCTF test has a relatively slow cooling rate than TS test. In FCTF tests, the interfacial residual stress had 
219 sufficient time to dissipate into a thick composite (sample C) coat, but was saturated in a thin buffer layer (sample B). The DLC TBC, the sample D, survived for 1143 cycles $(24,000$ EOH) without spallation of the top coat, showing a superior thermal fatigue resistance. The densified microstructure of the top coat was observed after the tests, showing fewer splat boundaries, crack coalescence, and growth compared with the as-coated microstructure.

Detailed microstructural comparison before and after FCTF tests is explained in section 3.4 with the results of the TS and JETS tests in Fig. 6. FCTF tests of sample D suggest that combining graded composite top coat and buffer layer is an effective strategy to improve the thermal fatigue resistance.

Delamination occurred within the top coat in the vicinity of the thermally grown oxide (TGO) layer in the samples A, B, and C, as shown in Fig. 3. The average thicknesses of each TGO layer in the sample $A, B, C$, and $D$ were $4.8,4.1,4.8$, and $7.3 \mu \mathrm{m}$, respectively. During FCTF tests, the development of TGO layer (black: $\alpha-\mathrm{Al}_{2} \mathrm{O}_{3}$ ) was observed at the interface of the top and bond coats in all samples, while the spinel composition (dark gray: $\mathrm{Cr}_{2} \mathrm{O}_{3}, \mathrm{NiAl}_{2} \mathrm{O}_{4}$, and other spinel structures, which are indicated with white arrows in Fig. 3) in the TGO layer developed in the samples $B, C$, and D, increasing the thickness with thermal exposure time. If the thickness of TGO layer is greater than $10 \mu \mathrm{m}$, the interface between TGO layer and top coat normally starts to be delaminated and shows a failure phenomenon. The formation mechanisms and composition of TGO layer have been well documented in literature $[22,23]$. As a result, an improvement of lifetime performance can be obtained in an actual TBC system, resulted from introducing a buffer layer and/or controlling the composition ratio, which can protect against oxidation of the bond coat and growth of a TGO layer. 
TS tests were performed for all samples, and the numbers of cycle-to-failure and surface status are summarized in Table 1. The SLC TBCs, the samples A and C, were fully delaminated within 10 and 14 cycles, respectively. On the other hand, the DLC TBCs with a buffer layer showed improved TS resistance. Delamination in the DLC TBCs with the single and double buffer layers occurred within 29 and 54 cycles, respectively. Consequentially, TS resistance can be effectively improved by introducing a buffer layer between the top and bond coats. Our results suggest that, during high cooling rate such as TS tests, buffer layer provides localized stress relief due to its high strain compliance. By introducing a buffer layer at the interface between the top and bond coats in the SLC TBC, the magnitude of the thermal and residual stresses can be diffused quickly, which is important for applications with relatively fast cooling/heating rates [24]. In particular, the thermal and residual stresses imposed on the sample D could be distributed into three layers by introducing a double buffer layer. Therefore, the DLC TBC with a double buffer layer shows the most improved TS resistance.

The cross-sectional microstructures of each TBC sample after TS tests are shown in

Fig. 4. The numbers of cycle-to-failure corresponding to each figure are shown inside each figure. The SLC TBCs, the samples A and C, were delaminated within just 14 cycles (4-10, and 7-14 cycles for the samples A and C, respectively), showing a thinner TGO layer than those in FCTF tests. This is due to the difference in thermal exposure time between the FCTF and TS tests. The DLC TBCs with a buffer layer, the samples B and D, delaminated in ranges of 13-29 and 46-54 cycles, respectively. There were full delamination in the sample B and partial delamination in the sample $\mathrm{D}$, with large horizontal cracks at the interface of the two buffer layers. Delamination in the samples B and D was created within the composite 
layer, due to the lower fracture toughness of LZO than that of 8YSZ. Also, the CTE mismatch between the bond coat and the LZO was reduced by introducing a buffer layer $[6,15]$. Therefore, the CTE difference should not be the only reason for delamination in LZO-based TBCs. Delamination can more easily occur in SLC TBCs because of the low fracture toughness of LZO and the high CTE mismatch. Failure in DLCTBCs was probably caused by a combination of its low fracture toughness and high stresses created by CTE mismatch. In TS tests, before water quenching, the temperature of all of the samples was about $1100{ }^{\circ} \mathrm{C}$. Comparing the LZO- and YSZ-based top coats, the bond coat and substrate have higher CTEs than the top coats, resulting in a fast volume shrinkage of the bond coat and the substrate during quenching sequence. In-plane compressive stresses and out-of-plane tensile stresses would be created, and small cracks and/or splat boundaries coalesced and propagated within the top coat near the interface. The partial delamination resulted from horizontal crack growth can prevent the top coat from catastrophic full delamination in actual application. However, the bond coats in all samples were degraded more in TS tests than in FCTF tests. In TS tests, the TGO layer consisted of $\mathrm{Al}_{2} \mathrm{O}_{3}$, which was easily cracked because of its low CTEs and brittleness in the water-quenching sequence [25]. The penetration of oxygen into the bond coat was difficult to prevent due to multiple cracks. On the other hand, development of a uniform and thick TGO layer prevented the permeation of oxygen in FCTF tests, resulting from the slower cooling sequence than in TS tests.

3.4 Jet engine thermal shock test and comparison

The numbers of cycle-to-failure and surface status in JETS tests are summarized in

Table 1. The microstructures of samples after JETS tests are shown in Fig. 5. The numbers of cycle-to-failure corresponding to each figure are shown inside each figure. The first 
delamination occurred in the SLC TBC with a 50:50 volume ratio (Fig. 5(A)) at an early stage of within 70 cycles (60-70 cycles) with catastrophic failure, and then the sample $\mathrm{C}$ with a 25:75 volume ratio (Fig. 5(C)) was delaminated in a range of 996-1022 cycles. As shown in Fig. 5, delamination happened near the interface between the bond and top coats in the SLC TBCs, showing similar fracture position and behavior with prior microstructures after cycles, showing a superior thermal stability.

In our previous work [26], the single-layer porous 8YSZ coating survived after 2000 cycles in the similar condition, while the double-layer coating with the porous 8YSZ buffer other coatings of the single-layer LZO coating and the double-layer coating with the dense 8YSZ buffer layer and the LZO top coat were fully delaminated within 25 and 885 cycles, respectively. As a result, the effects of the blended feedstock powder and buffer layers on improving thermal durability could be verified. During JETS tests, densification occurred from the surface of the top coat, resulting in generation of vertical cracks in the samples B and D (indicated with white arrows in Fig. 5), due to the disappearance of splat boundaries and pores. The growth and coalescence of horizontal cracks were observed at the interface between the buffer layer and the top coat in the sample B, and between the buffer layers in the sample D (indicated with black arrows in Fig. 5). This microstructure of the vertical cracked TBC system can enhance the thermal durability with improved strain compliance during operations [27]. As a result, the DLC TBCs with a buffer layer will show a superior thermal durability because of their vertical cracks. Similar to previous results of the FCTF and TS tests, the results of JETS tests indicated that the buffer layer could diffuse the stresses caused by CTE mismatch and improve the thermal durability of TBCs. On the other hand, 
interfacial stability was obtained without any evident growth of TGO scale, even compared with the as-coated microstructures in Fig. 2. This interfacial stability is the result of the thermal barrier's characteristics of low thermal conductivity material and relatively short thermal exposure time compared with the FCTF and TS tests.

Since the sample D demonstrated the best thermal durability among all samples, its microstructure and Vickers hardness of as-prepared, after the FCTF, TS, and JETS tests are summarized in Figs. 6 and 7, respectively. After FCTF tests, densification in the top coat and cracks within the buffer layer were observed, while the top coat after TS tests contained a large horizontal crack, which caused the partial delamination. From Fig. 6(C), the thickness of coating layer left after TS tests was about 200-250 $\mu \mathrm{m}$. The designed thickness of each buffer layer was $60 \mu \mathrm{m}$ and the total thickness of the double buffer layer formed was about $120 \mu \mathrm{m}$ (Fig. 1(D)). Therefore, it can be said that the top coat was partially delaminated, not fully delaminated. After TS tests, the temperatures in the substrate and bond coat are the same as with the top coat, resulting in horizontal cracks due to a rapid shrinkage of the substrate and the bond coat caused by a large CTE mismatch. However, JETS tests created vertical cracks (not shown in Fig. 6) by exposure of only the top coat surface to direct flame and horizontal cracks between the buffer layers.

Mechanical property, hardness measured using Vickers indentation, for the sample D is summarized in Fig. 7 and Table 2, before and after thermal exposure tests. The average imprint sizes of the as-prepared sample $D$ in Vickers indentation were 29.2, 35.2, and 28.7 um for the top coat, the buffer layer, and the bond coat, respectively. In general, there was an increase of $H$ values in the whole coating layer after thermal exposure tests, as shown in Table 2. The main reason for the increase is due to densification in the ceramic layer (buffer layer and top coat) and oxidation of the bond coat during thermal exposure. In the FCTF and 
339 TS tests, the increase of $H$ values in the buffer layer was less than for those of the top coat 340 (composite layer) because of the position of the buffer layer, where it can be protected from severe exposure. On the other hand, the increase in the top coat after TS tests was larger than that after FCTF tests, even though the thermal exposure time of TS tests was shorter than that of FCTF tests. A dense microstructure with coalescence of cracks rather than splat boundaries and tiny pores was achieved, which increased $H$ values. The $H$ values after JEST tests were significantly increased compared with those after the FCTF and TS tests. This is mainly due to the higher testing temperature and direct flame exposure in JETS tests. The surface of TBC is exposed to a high-temperature flame of $1400{ }^{\circ} \mathrm{C}$, resulting in a severe sintering effect. One other explainable mechanism is similar to the microstructure difference after the FCTF and TS tests, based on microstructure evolution.

Elastic modulus and hardness values measured using nano-indentation for the sample D is summarized in Fig. 8 and Table 2, before and after thermal exposure tests, except TS tests. The imprints after nano-indentation are shown in figure, indicating as numbers marked on the plot in the upper panel. The E and $H$ values measured using nano-indentation are increased with a similar trend with the $H$ values measured using Vickers indentation, indicating that the microstructure evolution supports the observed mechanical properties in cyclic thermal environments. The composite coatings with a double buffer layer can prevent a catastrophic failure with a superior thermal durability.

\section{Conclusions}

New architectures of $\mathrm{La}_{2} \mathrm{Zr}_{2} \mathrm{O}_{7}$ (LZO)-based TBC were designed using the blended LZO and 8YSZ feedstock powders and buffer layers, and prepared using APS method. The 362 microstructure, thermal durability, and mechanical properties were systematically 
investigated. The conclusions are summarized below:

(1) The DLC TBC with blended 50:50 volume ratio of LZO and 8YSZ powders and with a double buffer layer showed the most outstanding thermal durability among all samples in the FCTF, TS, and JETS tests. The improved thermal durability is due to the reduced thermal and residual stresses at the interfaces by decreasing CTE mismatch with the relatively continuous composition variation, and stress dissipation in the composite top coat by introducing the double buffer layer.

(2) For applications with relatively slow cooling rates similar to FCTF test, the thermal durability in the SLC TBCs is more effectively enhanced by controlling the composition ratio in the blended powder, which shows a better thermal durability than introducing a single buffer layer. This is due to the fact that, in FCTF tests, the interfacial residual stress has sufficient time to dissipate into a thick composite top coat, but is saturated in a thin buffer layer.

(3) For applications with relatively fast cooling rates similar to the TS and JETS tests, the thermal durability can be effectively improved by introducing a buffer layer than controlling the composition ratio in the composite top coat. Buffer layer provides fast localized stress relief due to its high strain compliance. By introducing a buffer layer at the interface between the top and bond coats, the thermal and residual stresses could be diffused quickly. The partial delamination of the top coat in the DLC TBC with a double buffer layer would be caused by the coalescence and growth of horizontal cracks. The vertical cracks in the DLC TBCs that were generated during JETS tests could release the thermal and residual stresses induced by CTE mismatch.

(4) The measured elastic modulus and hardness values were not significantly different in all samples, even though discontinuity was observed at the interface between the 
bond and top coats, no matter with or without the buffer layer. However, the continuity or discontinuity of mechanical properties, both elastic modulus and hardness values, at the interface between the top and bond coats was a negligible for lifetime performance of TBC systems in thermal exposure tests.

(5) Microstructure design through blending two feedstock powders and introducing a buffer layer enhanced thermal durability. The thermal durability of LZO-based TBC can be improved through combining the graded composite top coat and the buffer layer. This research can provide important microstructure design information for future advanced TBC design.

\section{Acknowledgments}

This work was supported by a National Research Foundation of Korea (NRF) grant funded by the Korean Government (2011-0030058), by Changwon National University in 2015-2016,by the Power Generation \&Electricity Delivery of the Korea Institute of Energy Technology Evaluation and Planning(KETEP) grants funded by the Korean Government Ministry of Trade, Industry and Energy (2013-101010-170C/2011T100200224),and by the United State Department of Energy (Grant No. DE-FE0008868, program manager: Richard Dunst).

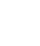
年 


\section{References}

411 [1] N.P. Padture, M. Gell, E.H. Jordan, Prog. Mater. Sci. 46 (2001) 505-553.

412 [2] D.R. Clarke, M. Oechsner, N.P. Padture, MRS Bulletin 37 (2012) 891-898.

413 [3] D. Clarke, C. Levi, Annu. Rev. Mater. Res. 33 (2003) 383-417.

414 [4] R.A. Miller, Surf. Coat. Technol. 30 (1987) 1-11.

415 [5] J.H. Zaat, A Quarter of a Century of Plasma Spraying, Annu. Rev. Mater. Sci. 13 (1983) 416 9-42.

417 [6] R. Vaßen, X. Cao, F. Tietz, D. Basu, D. Stöver, J. Am. Ceram. Soc. 83 (2000) 2023-2028.

418 [7] X.Q. Cao, R. Vaßen, D. Stöver, J. Eur. Ceram. Soc. 24 (2004) 1-10.

419 [8] J.D. Osorio, J.P. Hernandez-Ortiz, A. Torod, Ceram. Inter. 40 (2014) 4663-4671.

420 [9] Y.X. Zhao, L. Wang, J.S. Yang, D.C. Li, X.H. Zhong, H.Y. Zhao, F. Shao, S.Y. Tao, J. 421 Therm. Spray. Technol. 24 (2015) 338-347.

422 [10] W. Ma, D.E. Mack, R. Vaßen, D. Stöver, J. Am. Ceram. Soc. 91 (2008) 2630-2635.

423 [11] J. Hu, H. Zhao, S. Tao, X. Zhou, C. Ding, J. Eur. Ceram. Soc. 30 (2010) 799-804.

424 [12] R. Gadow, M. Lischka, Surf. Coat. Technol. 151 (2002) 392-399.

425 [13] H. Lehmann et al., J. Eur. Ceram. Soc. 86 (2003) 1338-1344.

426 [14] J. Zhang, 2015 NETL Crossing Cutting Research Review Meeting, Pittsburgh, PA, April $427 \quad 27-30(2015)$.

428 [15] G.K. Beshish, C.W. Florey, F.J. Worzala, W.J. Lenling, J. Therm. Spray Technol. 2 $429 \quad$ (1993) 35-38.

430 [16] J. Wu, X. Wei, N.P. Padture, P.G. Klemens, M. Gell, E. Garcia, P. Miranzo, M.I. Osendi, 431 J. Am. Ceram. Soc. 85 (2002) 3031-3035.

432 [17] R. Vaßen, F. Traeger, D. Stöver, Intern. J. App. Ceram. Technol. 1 (2004) 351-361.

433 [18] L. Wang et al., Ceram. Inter. 38 (2012) 3595-3606. 
434 [19] C. Wang et al., J. Alloys Comp. 649 (2015) 1182-1190.

435 [20] Y. Radin, T. Kontorovich, International Conference on Power Energy System 13 (2012)

$436 \quad 175-178$.

437 [21] X. Guo et al., Proceedings of the Energy Technologies and Carbon Dioxide Management 438 Sympositum, TMS 2016, Tennessee, USA, 151-160.

439 [22] O. Trunova, T. Beck, R. Herzog, R.W. Steinbrech, L. Singheiser, Surf. Coat. Technol. $440202(2008) 5027-5032$.

441 [23] K. Ma, J.M. Schoenung, Surf. Coat. Tech., 205 (2011), 5178-5185.

442 [24] W.G. Mao et al., Surf. Coat. Technol. 205 (2011) 3093-3102.

443 [25] General Cologne Re, Power Re-View, https://www.facworld.com/WebLib.NSF/

444 ObjectPreview/1Power2.pdf/\$File/Power2.pdf

445 [26] X. Guo, Z. Lu, Y.G. Jung, L. Li, J. Knapp, J. Zhang, Metal. Mater. Trans. 3E (2016) 6444670.

447 [27] E.A.G. Shillington, D.R. Clarke, Acta Mater. 47 (1999) 1297-1305.

448

449

450

451

452

453

454

455

456

457 
Table 1. Summary of the numbers of cycle-to-failure and surface status in each thermal exposure test

Table 2. Summary of elastic modulus and hardness values in DLC TBCs of 50:50 composition with a double buffer before and after thermal exposure tests

\section{Figure captions}

Fig. 1. Schematic diagram of microstructure design for the single- and double-layer TBCs:

SLC TBC of 50:50 composition without a buffer layer, (B) DLC TBC of 50:50 composition

with a buffer layer, (C) SLC TBC of 25:75 composition without a buffer layer, (D) DLC TBC of 50:50 composition with a double buffer layer.

Fig. 2. Cross-sectional microstructure, elastic modulus, and hardness of as-coated TBCs: (A)

SLC TBC of 50:50 composition without a buffer layer, (B) DLC TBC of 50:50 composition with a buffer layer, (C) SLC TBC of 25:75 composition without a buffer layer, (D) DLC TBC of 50:50 composition with a double buffer layer. Each number indicates microstructure and mechanical properties of elastic modulus and hardness, respectively. The dotted black, dotted blue, solid black, solid blue lines indicate the interfaces of the top and bond coats, the top coat and the first buffer layer, the top coat and the second buffer layer, and the second and first buffer layers, respectively

Fig. 3. Cross-sectional microstructure after FCTF tests: (A) SLC TBC of 50:50 composition without a buffer layer, (B) DLC TBC of 50:50 composition with a buffer layer, (C) SLC TBC of 25:75 composition without a buffer layer, (D) DLC TBC of 50:50 composition with a double buffer layer. The numbers of cycle-to-failure corresponding to each figure are shown inside each figure. Each number indicates the low- and high-magnified microstructures, and 
high-magnified interface microstructure, respectively. Arrows indicate the TGO layer of dark grey.

Fig. 4. Cross-sectional microstructure after TS tests: (A) SLC TBC of 50:50 composition without a buffer layer, (B) DLC TBC of 50:50 composition with a buffer layer, (C) SLC TBC of 25:75 composition without a buffer layer, (D) DLC TBC of 50:50 composition with a double buffer layer. Each number indicates the low- and high-magnified microstructures, respectively. The numbers of cycle-to-failure corresponding to each figure are shown inside each figure.

Fig. 5. Cross-sectional microstructure after JETS tests: (A) SLC TBC of 50:50 composition without a buffer layer, (B) DLC TBC of 50:50 composition with a buffer layer, (C) SLC TBC of 25:75 composition without a buffer layer, (D) DLC TBC of 50:50 composition with a double buffer layer. Each number indicates the low- and high-magnification microstructures, respectively. The white and black arrows indicate vertical cracks generated and horizontal cracks coalesced during JETS tests. The numbers of cycle-to-failure corresponding to each figure are shown inside each figure.

Fig. 6. Cross-sectional microstructure of DLC TBC of 50:50 composition with a double buffer layer before and after thermal exposure tests: (A) as-coated, (B) after FCTF test, (C) after TS test, and (D) after JETS test. Each number indicates the low- and high-magnification microstructures, respectively.

Fig. 7. Vickers hardness values of DLC TBCs of 50:50 composition with a double buffer layer before and after thermal exposure tests, measured using Vickers indentation. The imprints after Vickers indentation are shown in figure, indicating as numbers marked on the plot in the upper panel.

Fig. 8. Elastic modulus and hardness values of DLC TBCs of 50:50 composition with a 
506 double buffer layer before and after thermal exposure tests, measured using nano-indentation.

507 The imprints after nano-indentation are shown in figure, indicating as numbers marked on 508 the plot in the upper panel. 